\title{
Surveillance radiotoxicologique des travailleurs ; le dosage de la créatinine dans les urines
}

\author{
J. CAMARASA (*), J. CHALABREYSSE $\left({ }^{\star \star}\right)$, \\ F. TEULON $\left({ }^{\star \star}\right)$, E. RONGIER $(\star \star)$
}

\begin{abstract}
RÉSUMÉ
La surveillance radiotoxicologique des travailleurs exposés à des composés d'uranium pose des problèmes délicats d'interprétation des analyses d'urine. Pour effectuer les corrections nécessaires sur les échantillons, les auteurs rapportent le taux d'uranium excrété à la teneur en une substance provenant du métabolisme musculaire, la créatinine. Ils indiquent les avantages et les limites de cette méthode.
\end{abstract}

\section{ABSTRACT}

The radiotoxicological monitoring of uranium workers raises a number of problems in the interpretation of urinary analyses. In order to make the necessary corrections on the samples, the rate of excreted uranium is plotted vs creatinine levels. The advantages and limits of the method are stated.

\section{INTRODUCTION}

En médecine du travail et plus particulièrement en usine, la surveillance toxicologique systématique des travailleurs par l'analyse des urines ne peut se faire qu'à partir d'échantillons étant donné les difficultés pratiques de recueil de la totalité des urines de 24 heures; ce n'est que pour affiner un résultat anormal que le contrôle porte alors sur les urines de 24 heures. Mais, les résultats obtenus dans ces conditions n'ont qu'une valeur d'approximation en raison des nombreuses variations que peut subir l'élimination des composés urinaires durant le nycthémère et toute extrapolation des résultats fournis par un échantillon aux urines de 24 heures peut être entachée d'erreurs [2]. De nombreux auteurs ont essayé de les corriger en les rapportant à la créatinine urinaire [5-10].

Rappelons que la créatinine endogène est unc substance provenant du métabolisme musculaire. C'est la forme d'élimination de la créatinine dont elle dérive par déshydratation. Elle est éliminée uniquement par les urines à la suite d'une filtration glomulaire suivie d'une sécrétion négligeable

(*) Service médical, Péchiney-Ugine-Kuhlman, Comurhex, usine de Malvési, Narbonne, BP 70, 11101 Narbonne.

(**) Commissariat à l'énergie atomique, Département de protection, Service d'hygiène industrielle, BP 38, 26701 Pierrelatte Cedex. 
lorsque la créatininémie est normale [3-4]. La plupart des auteurs s'accordent pour reconnaître que :

- la créatinine urinaire est très stable pour un individu donné ;

- elle ne varie ni avec le débit urinaire ni avec les apports alimentaires ;

- elle est fonction de la masse musculaire et de la valeur de la fonction rénale ;

- les valeurs habituellement retenues sont les suivantes:

. 20 à $35 \mathrm{mg} / \mathrm{kg} / 24 \mathrm{~h}$ pour un homme, soit environ ........................

. 17 à $28 \mathrm{mg} / \mathrm{kg} / 24 \mathrm{~h}$ pour une femme, soit environ .........................

. ou environ (homme et femme) ...............

La correction des résultats à l'aide de la créatinine est basée sur le principe suivant : la concentration des constituants de l'urine varie au cours du nycthémère en fonction de la quantité de liquide ingérée, donc avec la diurèse. Or, les résultats d'analyse fournis par un échantillon ne tiennent pas compte de ces variations. Mais, étant donné que la quantité de créatinine excrétée dans les urines de $24 \mathrm{~h}$ est constante, la concentration urinaire de la créatinine dans un échantillon reflète bien le taux de concentration ou de dilution de l'échantillon urinaire. Ainsi, si l'on rapporte le taux de concentration du toxique au taux de créatinine urinaire, on peut apprécier avec une plus grande précision la quantité de toxique réellement excrétée.

Nous avons appliqué cette procédure aux travailleurs exposés à des composés d'uranium, que nous surveillons depuis de nombreuses années [1]. Ayant constaté certaines variations et observé quelques difficultés d'application, nous avons ressenti la nécessité de vérifier le bien-fondé de cette procédure. Nous rapportons ici nos observations.

\section{MATÉRIEL ET MÉTHOdES}

Notre expérimentation a suivi de très près la surveillance de routine mise en place dans les conditions normales de travail d'une usine traitant les composés uranifères (uranium naturel) [1].

Les dosages d'uranium et de créatinine ont été effectués par le Service d'hygiène industrielle du CEA à Pierrelatte, selon les méthodes suivantes:

- Uranium, par fluorimétrie : Les sels d'uranium sont fluorescents quand ils sont exposés aux rayonnements ultra-violets; cette fluorescence présente une sensibilité considérable lorsque les sels d'uranyle sont incorporés par fusion avec du fluorure de sodium et du carbonate de sodium ; elle est directement proportionnelle à la quantité d'uranium. 
- Créatinine, par colorimétrie à $510 \mathrm{~nm}$ du composé jaune-orangé formé en solution alcaline avec l'acide picrique. Les dosages sont effectués avec un appareil d'analyses automatiques LKB sur les urines diluées au $1 / 100^{e}$ dans l'eau distillée.

\section{II.1. Surveillance systématique par des échantillons d'urines}

Elle a porté durant l'année 1979 sur 117 salariés (hommes), tous postés, travaillant en fabrication, suivis régulièrement par la mesure d'uranium contenu dans les urines. On a prélevé tous les deux postes (15 jours) environ, un échantillon de $125 \mathrm{ml}$. Chaque flacon a été analysé après homogénéisation à l'acide nitrique. On a mesuré systématiquement pour chacun d'eux la créatinine et l'uranium. Les résultats ont été exprimés sous les trois formes suivantes:

- uranium urinaire en microgrammes par litre ;

- créatinine en grammes par litre d'urine ;

- uranium urinaire en microgrammes par gramme de créatinine.

Les différents résultats ont été rapportés sur des fiches personnelles (2 800 résultats environ pour 117 salariés). Pour chaque salarié, nous avons ainsi obtenu entre 20 et 30 analyses différentes qui nous ont permis, en particulier, d'observer et de suivre les variations de la créatinine urinaire.

Ces résultats n'étaient cependant pas satisfaisants puisqu'on pouvait leur reprocher d'être obtenus à partir d'échantillons non représentatifs.

\section{II.2. Surveillance par les urines de 24 heures}

Nous avons donc été amenés à faire les mêmes analyses sur des urines prélevées par neuf volontaires durant 24 heures à raison d'un prélèvement par mois.

Nous avons dosé, chaque fois, uranium et créatinine, les résultats étant exprimés ainsi :

- uranium total des urines de 24 heures, en microgrammes ;

- créatininurie des 24 heures, en grammes;

. uranium, en microgrammes par litre d'urine;

. uranium, en microgrammes par gramme de créatinine.

Parallèlement, nous avons fait recueillir les urines de 24 heures à trois volontaires travaillant dans un laboratoire extérieur à l'usine et non exposés à l'uranium.

\section{II.3. Excrétion nycthémérale de la créatinine}

Nous nous sommes adressés à trois volontaires postés, travaillant dans trois ateliers différents, représentatifs de l'usine ( $\mathrm{UF}_{4}, \mathrm{UO}_{3}$, et uranates).

Pour chacun d'eux, nous avons recueilli les urines de 24 heures à quatre reprises en suivant les rotations de postes: 
pour le poste de $4 \mathrm{~h}$ à $12 \mathrm{~h}$, nous avons recueilli les urines à partir de $4 \mathrm{~h}$ du matin ;

pour le poste de $12 \mathrm{~h}$ à $20 \mathrm{~h}$, nous avons recueilli les urines à partir de $12 \mathrm{~h}$;

pour le poste de $20 \mathrm{~h}$ à $4 \mathrm{~h}$, nous avons recueilli les urines à partir de $20 \mathrm{~h}$;

pour la période de repos, nous avons recueilli les urines après 24 heures d'arrêt de travail.

Pour chaque volontaire et pour chaque poste, nous avons ainsi recueilli entre 6 et 8 flacons d'urine. A chaque émission d'urine, le salarié urinait dans un nouveau flacon que nous analysions séparément.

\section{RÉSULTATS ET COMMENTAIRES}

\section{III.1. Variations observées dans les échantillons d'urine}

- 2800 dosages ont été effectués sur 117 salariés hommes.

. moyenne d'excrétion de la créatinine en $\mathrm{g} / \mathrm{l}$ : ............. 1,75 g/l

écart maximal entre les différents chiffres

d'excrétion .............................. $0,20 \mathrm{~g} / \mathrm{l}-4,68 \mathrm{~g} / \mathrm{l}$

On observe des variations importantes d'excrétion urinaire de la créatinine dans les échantillons.

\section{III.2. Variations observées dans les urines de 24 heures}

\section{Résultats globaux}

- 129 dosages ont été pratiqués sur les neuf volontaires travaillant en usine.

a) grammes/litre

moyenne d'excrétion de la créatinine, en $\mathrm{g} / \mathrm{l}: \ldots \ldots \ldots \ldots \ldots \ldots, 1,16 \mathrm{~g} / \mathrm{l}$

écart maximal entre les différentes valeurs

d'excrétion ................................... $0,45 \cdot 2, \mathrm{~g} / \mathrm{l}$

b) grammes/24 heures

moyenne d'excrétion de la créatinine, en g/24 $\mathrm{h}: \ldots \ldots \ldots 1,51 \mathrm{~g} / 24 \mathrm{~h}$.

écart maximal entre les différentes valeurs

d'excrétion ............................. $0,24 \cdot 2,84 \mathrm{~g} / 24 \mathrm{~h}$.

Résultats individuels

Ils sont présentés dans le tableau I. 
TABLEAU ।

Variations de l'excrétion urinaire de la créatinine dans les urines de $\mathbf{2 4}$ heures (les neuf premiers individus sont des volontaires travaillant en usine ; les trois derniers individus sont des volontaires travaillant en laboratoire)

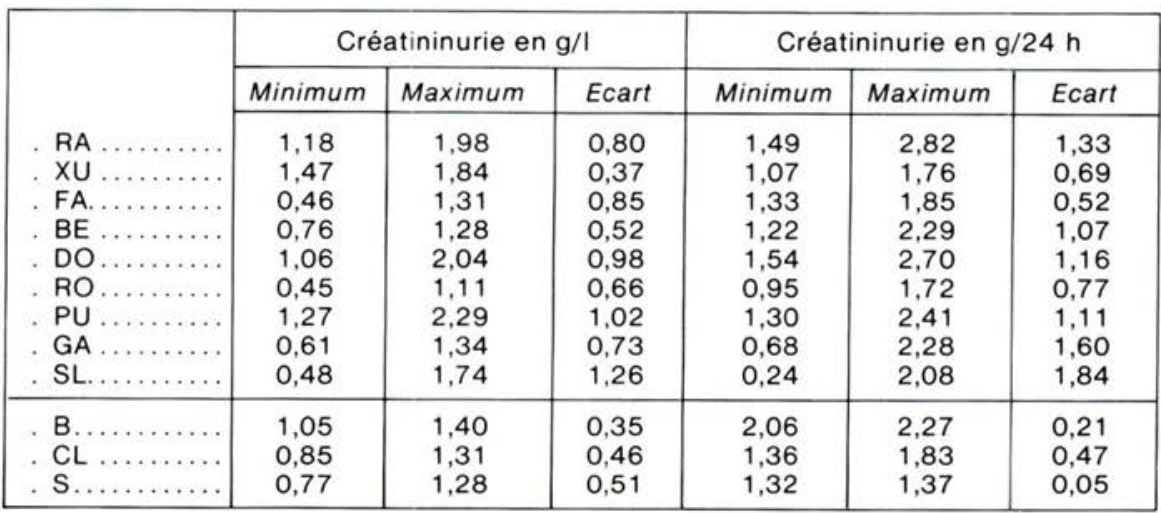

Ces écarts relevés sur chaque volontaire de l'usine portent sur un an environ (un prélèvement par mois). Ils correspondent aux valeurs minimales et maximales de créatininurie relevées pendant cette période pour chacun d'eux, soit 12 résultats environ. Pour le personnel du laboratoire, l'étude a porté sur 6 semaines (1 relevé par semaine), donc 6 résultats pour chacun d'eux.

On peut faire les observations suivantes:

- les moyennes d'excrétion sont comparables à celles relevées dans la littérature ;

- il existe des variations plus ou moins importantes dans les valeurs de créatinine de chaque volontaire (usine et laboratoire).

On peut remarquer que, pour le personnel de laboratoire, les variations relevées dans les valeurs d'excrétion de la créatinine en grammes par litre ou en grammes par 24 heures, sont relativement faibles et correspondent bien aux données de la littérature : $0,47 \mathrm{~g}$ de créatinine par 24 heures est l'écart le plus important. Par contre, pour les ouvriers de l'usine, la créatininurie varie très souvent, chez un même individu, suivant la date des prélèvements, dans des limites nettement plus importantes: $1,84 \mathrm{~g}$ de créatinine par 24 heures est l'écart le plus important. Le personnel de laboratoire aurait-il mieux recueilli les urines que celui de l'usine, compte tenu des difficultés rencontrées en usine dans la pratique courante ?... Nous ne le pensons pas, car nous avions affaire à des volontaires revus souvent, bien motivés et avertis. Nous pensons aussi que la répétition des prélèvements et la lecture des graphiques étalés sur une année permettraient de corriger d'éventuelles erreurs.

\section{III.3. Variations dans l'excrétion nycthémérale}

Les résultats sont consignés dans le tableau II. 
TABLEAU ॥

Variations de l'excrétion urinaire de la créatinine au cours du nycthémère (Résultats exprimés en $\mathrm{g} / \mathrm{l}$ sur des échantillons)

\begin{tabular}{|c|c|c|c|c|c|c|c|c|}
\hline \multirow{2}{*}{ Sujet } & \multicolumn{2}{|c|}{ Poste de nuit } & \multicolumn{2}{c|}{ Poste d'après-midi } & \multicolumn{2}{c|}{ Poste de matinée } & \multicolumn{2}{c|}{ Repos } \\
\cline { 2 - 9 } & Minimum & Maximum & Minimum & Maximum & Minimum & Maximum & Minimum & Maximum \\
\hline DO & 0,54 & 2,16 & 1,05 & 3,21 & 0,71 & 2,80 & 1,14 & 2,11 \\
\hline BE & 0,15 & 1,44 & 0,85 & 2,27 & 0,22 & 1,05 & 0,49 & 1,83 \\
\hline FA & 0,18 & 1,04 & 0,76 & 1,76 & 0,21 & 1,12 & 0,24 & 1,14 \\
\hline
\end{tabular}

On constate ainsi que les écarts peuvent aller du simple au quintuple : ils sont donc importants. La figure 1 est un exemple de courbes obtenues à partir des différents résultats pour chaque poste de travail. Nous avons pris le plus significatif ; il rend bien compte des variations de la créatinine en $\mathrm{g} / \mathrm{l}$ et du pic d'excrétion urinaire correspondant à la fin de poste :

D'une manière générale, en effet, on retrouve sur les différents graphiques un pic (en relation d'ailleurs avec celui du toxique urinaire) se

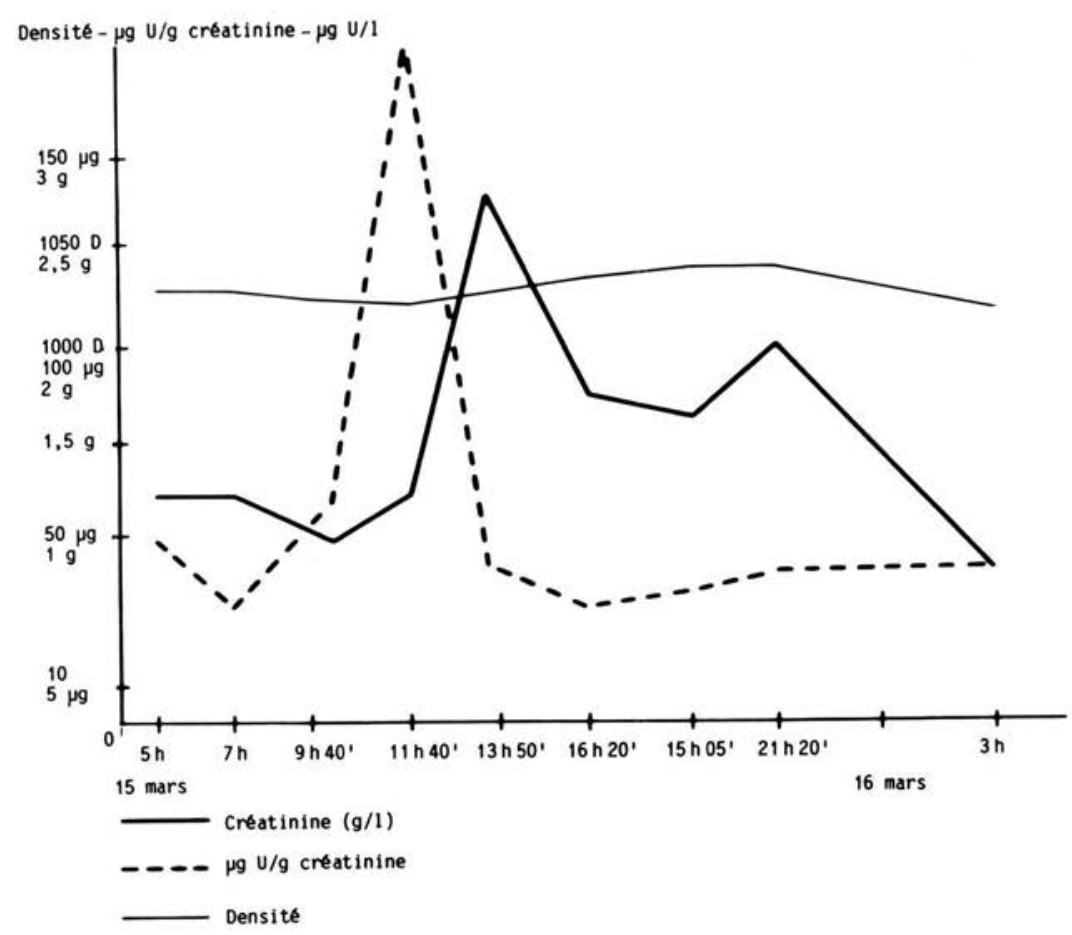

Fig. 1. - Excrétion nycthémérale de la créatinine. 
situant en fin de poste avec retour rapide au chiffre antérieur dans les heures qui suivent. Ce pic d'excrétion urinaire paraît correspondre à la période de travail, donc d'activité musculaire plus grande.

\section{III.4. Rôle de l'exercice musculaire}

Les écarts observés dans l'excrétion urinaire de la créatinine pour les échantillons de 24 heures (volontaires en usine et volontaires en laboratoire) sont liés à l'activité musculaire. On sait que la créatinine urinaire est fonction de la masse musculaire, donc de l'activité physique plus ou moins importante de l'individu.

Les données publiées dans la littérature indiquant que la créatinine urinaire est constante pour un individu donné ont été obtenues sur des sujets alités ou.hospitalisés. Durant l'activité professionnelle, le sujet exerce des efforts musculaires, entraînant des variations relatives de son excrétion urinaire en créatinine. Les différences observées entre travailleur en usine et travailleur en laboratoire sont dues au fait que le personnel de laboratoire a une activité physique moins importante que celui d'une usine. Ceci a pu être vérifié chez un volontaire travaillant en fabrication en usine, puis ayant cessé toute activité professionnelle après son départ à la retraite : les écarts constatés durant l'activité professionnelle ont disparu après cessation de l'activité.

\section{CONCLUSION}

\section{IV.1. Validité et limites de la créatininurie}

En milieu de travail, contrairement à ce que l'on était en droit de penser, l'excrétion urinaire de la créatinine, pour un même sujet, n'a pas la stabilité que lui reconnaissait la littérature : elle peut varier, non seulement au cours du nycthémère lorsqu'on étudie l'excrétion sur une courte période de 24 heures, mais aussi suivant la date des prélèvements lorsque ceux-ci sont espacés dans le temps.

Ces variations, tributaires assurément de la fonction rénale, sont probablement expliquées en partie par l'activité musculaire due au travail. C'est ainsi que le personnel au repos ou à activité physique minime a une excrétion relativement stable de la créatinine qui, par contre, augmente pendant un travail physique actif pour diminuer et redevenir stable pendant la période de repos physique.

En raison de ces variations, la créatininurie ne paraît pas avoir la valeur de référence absolue qu'on pourrait lui attribuer et on est toujours en droit de penser que la valeur mesurée dans un échantillon d'urine pour un toxique, même si elle est corrigée par la créatinine urinaire, ne représente pas l'exacte réalité de l'excrétion urinaire. 


\section{IV.2. Conduite à tenir en médecine du travail}

Dans notre usine, où les travailleurs sont suivis notamment par des contrôles de l'excrétion de l'uranium dans les urines [1], nous continuons à pratiquer le dosage de la créatinine malgré les imperfections et les incertitudes liées à cette procédure. Les résultats sont exprimés ainsi : uranium en microgrammes par litre d'urine, uranium en microgrammes par gramme de créatinine. Les valeurs ainsi obtenues sont reportées sur des graphiques qui transcrivent les résultats d'analyses espacés de quinze jours ou d'un mois. Ils représentent donc imparfaitement ce qui s'est passé dans les intervalles de temps. Cependant, comme nous recherchons une imprégnation générale du sujet en uranium plutôt qu'un seuil à ne pas dépasser, ce genre d'interprétation nous paraît répondre le mieux à cette demande et permet, en outre, de corriger les erreurs ponctuelles dues à l'échantillonnage.

Le suivi régulier, chez un travailleur, des courbes d'élimination de l'uranium exprimée en microgrammes par litre d'urine et celles de l'élimination de l'uranium exprimée en microgrammes par gramme de créatinine montre un parallélisme évident entre les deux avec une correction dans le sens de la régularité d'élimination pour la deuxième permettant d'atténuer les grandes variations apparaissant sous la forme de pics d'élimination urinaire. On peut ainsi mieux corriger les erreurs d'appréciation.

Le dosage simultané de la créatinine dans l'urine permet, en outre, d'éliminer des "faux" échantillons d'urine (rajout volontaire d'eau du robinet à une miction insuffisante par le travailleur lui-même) ou de détecter des insuffisances rénales, ce qui est particulièrement intéressant pour des toxiques rénaux tels que l'uranium.

\section{IV.3. Autres possibilités de correction}

Depuis un an environ, nous avons rapporté l'excrétion urinaire de l'uranium à la densité des échantillons d'urines (valeur moyenne retenue $=1025$ ). Nos observations ne sont pas en nombre suffisant pour pouvoir conclure, mais il ne nous semble pas que la correction ainsi obtenue soit meilleure que celle avec la créatinine.

\section{BIBLIOGRAPHIE}

[1] CAMARASA J., CHALABREYSSE J.- Contribution à la surveillance du personnel exposé à des composés d'uranium non transférables. Radioprotection, 1980, 15, 3-8.

[2] CURTIS G., FOGEL M. - Excrétion de la créatinine : variation nycthémérale et variabilité des mesures effectuées sur une journée ou une partie de la journée. Etude méthodologique dans la recherche psycho-endocrinienne. Psychosom. Med. USA, $1970,32,337-350$.

[3] GRAFMEYER D., COHEN R., BADINAND A. - Microdétermination de la créatinine dans les liquides biologiques par des méthodes cinétiques (Comparaison avec les méthodes en flux continu). Ann. Biol. Clin., 1976, 34, 283-289. 
[4] GRAFNETTER D. et Coll. - Dosage spécifique de la créatinine par la réaction de Jaffe, d'après la méthode de Slot. Pharmacien Biolog., 1971, n 71, suppl.

[5] GUIDICE S., NARDELOTTO O. - Comparison between the elimination of urine "in toto", dry substances and creatinine. Venise : Montedison-Marghera, Medical Department, April 1980.

[6] GÜRSON C.T., SANER G. - Urinary chromium excretion, diurnal changes, and relationship to creatinine excretion in healthy and sick individuals of different ages. Am. J. Clin. Nutrit. 1978, 31, 1162-1166.

[7] HOUSSET H., BOUCKER M., GERVAIS P. et al. - Expression des résultats des dosages de l'acide delta-aminolévulinique urinaire en fonction de la créatininurie dans l'exposition professionnelle au plomb. Arch. Mal. Prof. 1977, 38, 303-313.

[8] JACKSON S. - Creatinine in urine as an index of urinary excretion rate. Health Physics, 1966, 12, 843-850

[9] LAURENT B. - Résultats des examens biologiques exprimés par gramme de créatinine chez les enfants. Pharmacien biologiste, 1977, 11, 503-505.

[10] MEGEMONT Ch., ROSAY J., JACQ J. - A propos du dosage de la créatinine sur les prélèvements des urines des 24 heures destinées aux examens de radiotoxicologie : intérêt pour l'interprétation des résultats. Arch. Mal. Prof., 1971, $32,128$. 